\title{
Is the ISNT rule violated in early primary open-angle glaucoma-a scanning laser tomography study
}

R Sihota, G Srinivasan, T Dada, V Gupta, D Ghate and A Sharma

\section{Abstract}

Aims To evaluate the relevance of the ISNT rule with reference to the optic nerve head, in differentiating normal and early glaucoma eyes and neuroretinal rim (NRR) area ratios as measures of glaucomatous optic neuropathy by confocal scanning laser ophthalmoscopy (Heidelberg retina tomography (HRT) II). Methods The study included 136 control eyes and 63 eyes of early primary open-angle glaucoma. Each patient underwent a complete ophthalmic examination, HRT II (software 2.01) and achromatic automated perimetry using the Humphrey field analyzer Full threshold program 30-2 or 24-2. Topographic HRT parameters (disc area and rim area) were compared between the groups. To assess the statistical significance of differences between the study groups, the Student's $t$-test was used.

Results The ISNT rule was applicable in $71 \%$ of normal eyes and $68 \%$ of early glaucoma eyes. The superior to inferior area ratio was $0.96 \pm 0.01$ in the normal group and $0.90 \pm 0.02$ in the glaucoma group. There was a loss of approximately a quarter of the NRR in the inferotemporal and superotemporal quadrants. The inferonasal sector showed the least loss of NRR (4.34\%).

Conclusion The inferior NRR is marginally wider than the superior NRR in about $2 / 3$ of normal eyes, but could not be clinically appreciated in many of these. The characteristic configuration of a normal optic disc with the rim width being greatest in the inferior disc region followed by the superior disc region was maintained even in most patients with early glaucoma. Eye (2008) 22, 819-824; doi:10.1038/sj.eye.6702798; published online 13 April 2007
Keywords: detection; glaucoma; open angle; neuroretinal rim; scanning laser ophthalmoscopy

\section{Introduction}

The normal optic disc is slightly vertically oval. The neuroretinal rim (NRR) of the optic nerve head is the intrapapillary extension of the optic nerve fibres. As glaucoma leads to a loss of NRR, measurement of this is critical in the morphometric analysis of the optic disc in glaucoma. ${ }^{1,2}$ The NRR, which is one of the main parameters in the diagnosis of glaucomatous optic neuropathy, can be defined by its area, ${ }^{1,2}$ volume above a reference level, shape and pallor.

In normal discs, the NRR is said to have a characteristic configuration with the rim width being the thickest in the inferior region, followed by the superior sector, the nasal area, and finally the temporal sector (ISNT-rule). ${ }^{3}$ Glaucoma is characterized by a progressive thinning of the NRR, which while occurring in all sectors of the optic disc, is said to show a preferential predilection for the inferior pole, thus leading to a loss of the physiological rim shape so that the inferotemporal rim is no longer the thickest. ${ }^{4-7}$

The purpose of the present study is to evaluate whether the ISNT rule as assessed on Confocal scanning laser tomography of the optic disc is applicable in Indian eyes and also to evaluate if the pattern of NRR area loss as quantified by the confocal scanning laser tomograph, Heidelberg retina tomography (HRT) II is predictive of early primary open-angle glaucoma (POAG).
Dr Rajendra Prasad Centre for Ophthalmic Sciences, All India Institute of Medica Sciences, New Delhi, India

Correspondence: R Sihota, Head of Glaucoma Service, Dr Rajendra Prasad Centre for Ophthalmic Sciences, New Delhi 110029, India

Tel: + 91111686485165 ; Fax: + 911126588919 E-mail: rjsihota@hotmail.com

Received: 22 February 2006 Accepted in revised form: 16 February 2007 Published online: 13 April 2007

Paper previously presented at the Glaucoma Society of India Conference 18 December 2005 


\section{Patients and methods}

A total of 63 patients with POAG and 136 normal controls $>40$ years of age were included in this retrospective study (Demographic data in Table 1). All patients were Asians between 40 and 75 years of age of North Indian origin, had visual acuity 20/40 or better, refractive error not exceeding 6.0D sphere and no prior ocular surgery. All subjects underwent a complete ophthalmic examination, including slit lamp biomicroscopy and confocal scanning laser tomography HRT II. Achromatic automated perimetry was performed with the Humphrey Field Analyzer using the 30-2 or 24-2 Full Threshold program.

Informed consent was obtained from all patients.

Only one randomly selected eye per control and patient was statistically analysed.

The criteria for the diagnosis of POAG were an increased IOP (measurements $21 \mathrm{~mm} \mathrm{Hg}$ or more by Goldmann applanation tonometry), open anterior chamber angle and typical glaucomatous changes of the optic nerve head on examination with a $+90 \mathrm{D}$ lens - vertical cup disc ratio $\geqslant 0.7: 1$, cup disc asymmetry of $\geqslant 0.2$, pallor, thinning or notching of the NRR, optic disc haemorrhage, retinal nerve fibre layer defects and glaucomatous visual field defects. All patients had visual field changes of an abnormal glaucoma hemifield test, an MD 0 to $-6 \mathrm{~dB}$, fewer than 18 points depressed below the $5 \%$ probability level and fewer than 10 points below the $P<1 \%$ level and no point in the central $5^{\circ}$ with a sensitivity of less than $15 \mathrm{~dB}$ (Hodapp classification for early glaucomatous field loss). ${ }^{8}$ The defect had to be shown in at least two visual fields repeated within a span of 6 months. None of the patients had any other ocular disease. All patients with retinal or neurological diseases were excluded from the study.

Normal controls had no history of ocular disease, an IOP of less than $21 \mathrm{~mm} \mathrm{Hg}$ measured by Goldmann applanation tonometry, normal disc, and normal perimetry. Absence of glaucomatous optic neuropathy was defined as a vertical cup-disc asymmetry of 0.2 or less and without any of the typical glaucomatous

Table 1 Demographic data of glaucoma group and control group

\begin{tabular}{lccc}
\hline Patient demographics & Control group & Glaucoma group & P-value \\
\hline $\begin{array}{l}\text { Number } \\
\text { Age (yrs), mean } \pm \text { SD }\end{array}$ & $43.13 \pm 13.0$ & $45.52 \pm 15.0$ & 0.25 \\
(range) & & & \\
Gender & $40 \mathrm{M} 23 \mathrm{~F}$ & $81 \mathrm{M} 55 \mathrm{~F}$ & 0.59 \\
Disc area $\left(\mathrm{mm}^{2}\right)$ & $2.40 \pm 0.04$ & $2.51 \pm 0.06$ & 0.18 \\
\hline
\end{tabular}

changes in the disc and peripapillary retina. Normal visual field indices were defined as a mean defect and corrected SD within $95 \%$ confidence limits and a glaucoma hemifield test within normal limits.

Confocal scanning laser ophthalmoscopy using the HRT II (version 2.01) was performed in a standardized manner by one experienced, masked examiner, who checked the disc outline in all cases. This technique has been described in detail elsewhere. ${ }^{9,10}$ Disc area was defined as the total area within the contour line. Rim area was defined as the area within the contour line and above the reference plane. HRT II assessment of disc area and rim area parameters was analysed. The sum of the rim areas in the superotemporal and superonasal region as well as the inferotemporal and inferonasal region was calculated. The values obtained for the superior and inferior rim areas, respectively, were compared with the temporal rim area value to assess the ISNT rule.

To assess the statistical significance of differences between the study groups, the Student's $t$-test was used. Correlation analyses used the Pearson's correlation coefficient. A P-value of 0.05 or less was considered statistically significant. All statistical analyses were performed using SPSSWIN 10 software.

\section{Results}

In $96(71 \%)$ control eyes, the inferior rim area was the largest followed by the superior rim area and the temporal rim area. In patients with early glaucoma also this pattern was seen in $43(68 \%)$. The superior by inferior ratio was less than 1.00 in $93(69 \%)$ in the control group and in 49 (78\%) in the glaucoma group. This was not statistically significant between the groups. The mean superior to inferior rim ratio was $0.96 \pm 0.01$ in the control group and $0.90 \pm 0.02$ in the early glaucoma group, respectively $(P=0.02)$. In the remaining glaucoma eyes, the superior rim was the largest followed by the inferior and temporal rim.

The NRR area in the superotemporal, inferotemporal, superonasal, inferonasal and temporal disc sectors were $0.21 \mathrm{~mm}^{2}( \pm 0.05), 0.23 \mathrm{~mm}^{2}( \pm 0.06), 0.25 \mathrm{~mm}^{2}( \pm 0.05)$, $0.26 \mathrm{~mm}^{2}( \pm 0.05)$ and $0.28 \mathrm{~mm}^{2}( \pm 0.08)$ in the normal group and $0.17 \mathrm{~mm}^{2}( \pm 0.06), 0.19 \mathrm{~mm}^{2}( \pm 0.06), 0.21 \mathrm{~mm}^{2}$ $( \pm 0.07), 0.25 \mathrm{~mm}^{2}( \pm 0.06)$ and $0.23 \mathrm{~mm}^{2}( \pm 0.01)$ in the early glaucoma group, respectively (Table 2; Figure 1). There was a statistically significant difference in all the values between the groups $(P=0.00,0.001,0.00,0.002$ and 0.001 respectively).

Although the inferior rim area was the largest in both the groups, the inferonasal area was found to be larger than the inferotemporal sector in both. The inferonasal and inferotemporal rim areas in the control group were $0.26 \mathrm{~mm}^{2}( \pm 0.05)$ and $0.23 \mathrm{~mm}^{2}( \pm 0.06)$, respectively and 
Table 2 Comparison of NRR areas and ratios in glaucoma and control group

\begin{tabular}{|c|c|c|c|c|}
\hline Neuroretinal rim sector & $\begin{array}{l}\text { Early glaucoma } \\
\{\text { Mean }( \pm S D)\}\end{array}$ & $\begin{array}{c}\text { Control eyes } \\
\{\text { Mean }( \pm S D)\}\end{array}$ & $\%$ Difference b/w groups & P-value \\
\hline 1. Superotemporal (ST) area & $0.17(0.06)$ & $0.21(0.05)$ & 23.5 & 0.000 \\
\hline 2. Inferotemporal (IT) area & $0.19(0.06)$ & $0.23(0.06)$ & 21.05 & 0.001 \\
\hline 3. Superonasal (SN) area & $0.21(0.07)$ & $0.25(0.05)$ & 19.04 & 0.000 \\
\hline 4. Inferonasal (IN) area & $0.25(0.06)$ & $0.26(0.05)$ & 4.34 & 0.002 \\
\hline 5. Temporal $(\mathrm{T})$ area & $0.23(0.01)$ & $0.28(0.08)$ & 21.73 & 0.001 \\
\hline 6. Disc area & $2.51(0.06)$ & $2.40(0.04)$ & 4.4 & 0.08 \\
\hline 7. ST/IT ratio & $0.98(0.04)$ & $0.98(0.02)$ & 0 & 0.98 \\
\hline 8. $\mathrm{SN} / \mathrm{IN}$ ratio & $0.86(0.02)$ & $0.96(0.01)$ & 11.62 & 0.001 \\
\hline 9. IT/IN ratio & $0.87(0.14)$ & $0.79(0.18)$ & 9.30 & 0.003 \\
\hline 10. S/I ratio & $0.90(0.02)$ & $0.96(0.01)$ & 6.60 & 0.02 \\
\hline 11. $\mathrm{S} / \mathrm{T}$ ratio & $1.90(0.09)$ & $1.79(0.05)$ & 4.70 & 0.35 \\
\hline 12. $\mathrm{I} / \mathrm{T}$ ratio & $2.19(0.10)$ & $1.88(0.05)$ & 14.15 & 0.03 \\
\hline
\end{tabular}

Abbreviations: I, inferior; $S$, superior.

In rows $1-5$, unit of measurement is $\mathrm{mm}^{2}$ ).

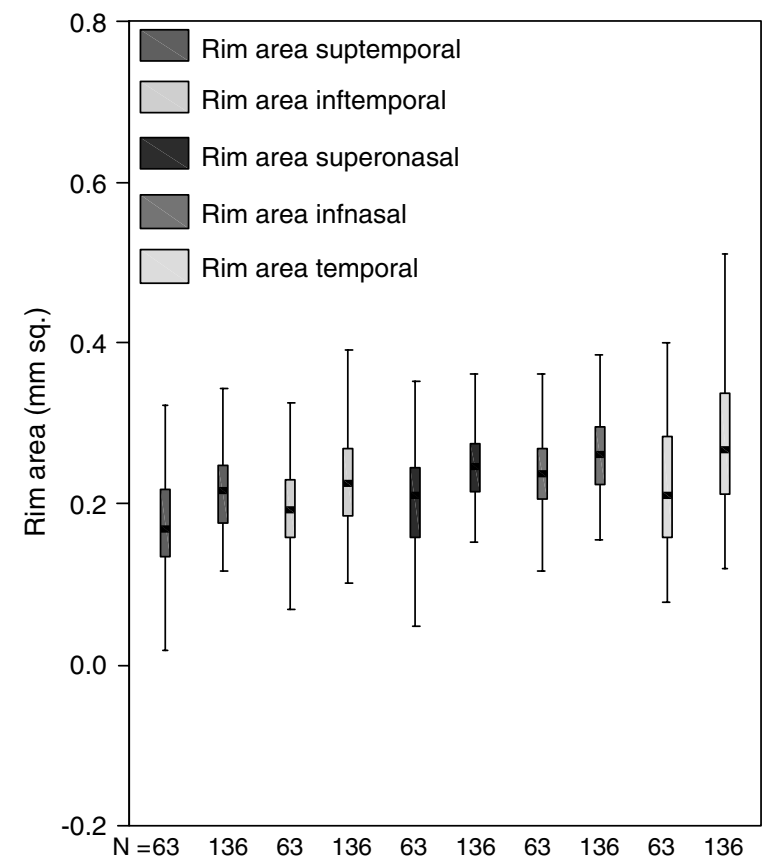

Figure 1 Rim areas of individual sectors in control $(n=136)$ and early glaucoma $(n=63)$ group.

$0.25 \mathrm{~mm}^{2}( \pm 0.06)$ and $0.19 \mathrm{~mm}^{2}( \pm 0.06)$ in the glaucoma group, respectively. The inferonasal sector showed the least change $(4.34 \%)$. Similarly, the superonasal rim area was larger than the superotemporal rim area in both the groups (Table 2).

The ratios of the rim areas of the superotemporal to inferotemporal sectors were found to be $0.98( \pm 0.02)$ and $0.98( \pm 0.04)$ in the control group and the glaucoma group, respectively ( $P=0.98$; Table 2 ; Figure 2$)$. The ratio of the superonasal to inferonasal rim sectors was 0.96 $( \pm 0.01)$ and $0.86( \pm 0.02)$ between the groups $(P=0.001)$, with the control group showing a significantly higher

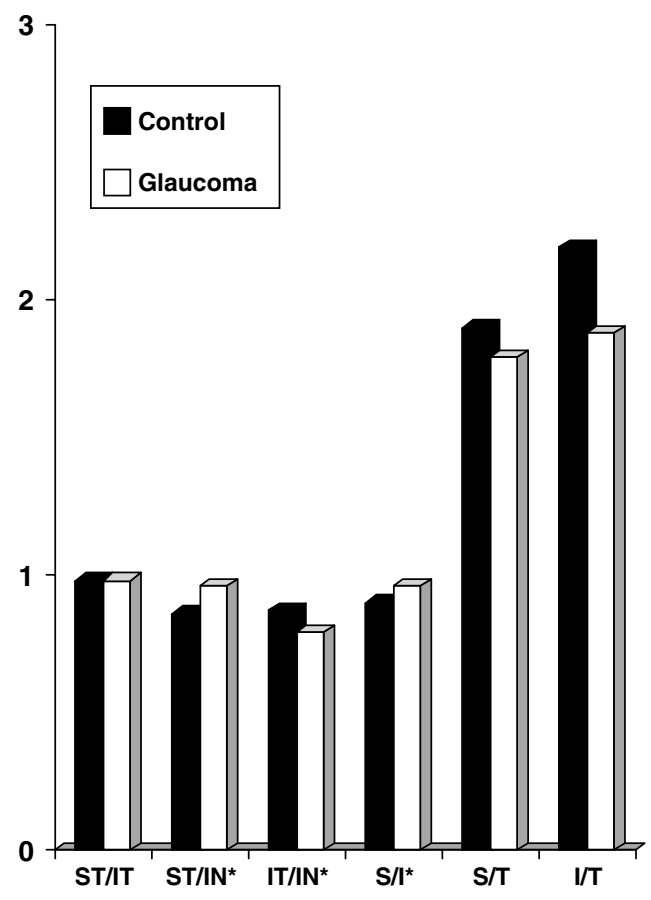

Figure 2 Ratio of rim areas between the two groups (*ratios with $P<0.05)$

ratio. The ratios of the rim areas of the inferotemporal to inferonasal sector were $0.87( \pm 0.14)$ and $0.79( \pm 0.18)$ in the control group and glaucoma group respectively $(P=0.003)$.

While the ratio of the superior to temporal sector was not found to be significantly different $(P=0.35)$ between the groups $(1.79( \pm 0.05)$ in control group and 1.90 $( \pm 0.09)$ in the glaucoma group), the ratio of the inferior to temporal sectors was $1.88 \pm 0.05$ in the control group and $2.19 \pm 0.10$ in the glaucoma group $(P=0.03)$.

The disc area was $2.40 \pm 0.04 \mathrm{~mm}^{2}$ in the control group and $2.51 \pm 0.06 \mathrm{~mm}^{2}$ in the glaucoma group $(P=0.18)$. 
Rim areas in the individual sectors in the present analyses were statistically independent of optic disc size, except for the inferonasal rim area. $(r=0.224$ at $P<0.001)$

Despite the fact that the NRR area in all the sectors in the glaucoma group was significantly less than in the normal subjects (Table 2), there was a very poor sensitivity when only this parameter was used to diagnose glaucoma. Among the rim areas and ratios analysed, the ROC curves of the superior/temporal rim ratio showed the highest predictability to differentiate early glaucoma from normal subjects (Table 3 ).

Analysis of the field showed superior field defects in 25 patients (39.6\%) (superonasal field defects in 16 patients and superotemporal defects in 09 patients). Inferior field defects were seen in 38 patients $(60.3 \%)$ (inferonasal field defects in 28 patients and inferotemporal defects in 10).

\section{Discussion}

Control eyes in our study showed that the inferonasal disc sector was largest, followed by the temporal and superonasal sectors. Taking the inferotemporal and inferonasal sectors together, they were larger than the superotemporal and superonasal sectors together, with a superior to inferior rim area ratio of $0.96 \pm 0.01$. Seventy-one percent of our control eyes supported the concept of the ISNT rule ${ }^{3}$ mathematically by the HRT II evaluation of the rim areas, wherein the rim width is broadest in the inferior disc region, followed by the superior disc sector, the nasal disc sector and finally the temporal disc sector. However, this difference was subtle and thus may be difficult to appreciate clinically.

In early stages of glaucoma, the NRR area is said to be preferentially lost in the inferior and superior optic disc

Table 3 ROC curves for different parameters

\begin{tabular}{ll}
\hline & AUC \\
\hline Neuroretinal rim area & \\
Superotemporal & 30 \\
Inferotemporal & 33 \\
Superonasal & 31 \\
Inferonasal & 38 \\
Temporal & 32 \\
& \\
Ratio of sectors & \\
ST/IT & 45 \\
SN/IN & 36 \\
S/I & 39 \\
S/T & 48 \\
I/T & 56 \\
IT/IN & 38 \\
Disc area & 56 \\
\hline
\end{tabular}

sectors and the rim configuration is said to change to one in which the rim width is more or less equal in all parts of the optic disc. ${ }^{7}$ This should lead to a loss of applicability of the ISNT rule in glaucomatous eyes. This has been reported by Jonas et al, ${ }^{11}$ who found that abnormally low rim inferior to temporal and superior to temporal rim width ratios due to this preferential loss of NRR can indicate glaucomatous damage in some ocular hypertensive eyes. The optic nerve head variables with the highest predictive value to differentiate between normal eyes and eyes with increased intraocular pressure and normal visual fields, are the shape of the NRR and visibility of the nerve fibre layer. ${ }^{12}$ However, even in our early glaucoma eyes $68 \%$ were found to retain the same NRR area configuration as is seen in control eyes with the ISNT rule, suggesting that the loss of NRR in most early glaucoma subjects (68\%) occurred in a diffuse manner so that they retained the same NRR area configuration as in normal subjects $(71 \%)$, with the inferior rim area being larger than the superior followed by the temporal rim area. This is in agreement with what we see in clinical practice, where we have not found the ISNT rule useful in detecting early glaucoma. The nasal horizontal optic disc sector has been excluded in the present study as it has been seen that the nasal sector is the last to be affected in glaucoma and our study was done in subjects with early glaucoma. ${ }^{13}$

In our study, the disc area in the control group $\left(2.40 \pm 0.04 \mathrm{~mm}^{2}\right)$ was larger than that reported in other studies $(1.99 \pm 0.52,1.87 \pm 0.45)^{14,15}$ but this can be explained by racial differences. Comparable larger disc dimensions in the normal population have also been reported in an earlier study from our centre. ${ }^{16}$ It is believed that the pattern of NRR loss depends on the type of glaucoma. In high-pressure glaucoma, characteristically seen in patients with juvenile open-angle glaucoma, the NRR is said to show a diffuse concentric loss, ${ }^{17}$ while the glaucomatous shape of the NRR is said to be better demonstrated in eyes with focal normal pressure glaucoma because they have localized defects in the temporal inferior and temporal superior sectors. ${ }^{18}$ The mean superior to inferior rim ratio dropped to $0.90( \pm 0.02)$ in our early glaucoma group, suggesting a comparatively greater loss of NRR superiorly. While this finding may indicate a greater NRR loss superiorly, it may also be reflective of a selection bias of this particular study population. But this does not take away from the fact that even if this slight loss in the NRR superiorly on quantitative evaluation by HRT II were to be present inferiorly, as we would expect, it would be seen clinically as an almost equal thickness of the superior and inferior rim, which may be difficult to pick up on subjective stereoscopic evaluation (Figure 3). So in Indian eyes, it would probably not be prudent to 
look for NRR loss in the inferotemporal rim sector as an indicator of early glaucoma. While the importance of the inferotemporal disc sectors in the diagnosis of glaucoma has been shown in numerous studies, ${ }^{19,20}$ Mardin et al ${ }^{21}$ found that the area and volume of the optic rim and cup and cup-shape measure showed higher sensitivities in the superior than the measurements in the inferior sector because of lesser contribution of the vessels to the rim in that sector. In our study, we found a percentage difference of NRR loss of $23.5 \%$ for the superotemporal rim area, $21.05 \%$ for the inferotemporal sector, $19.04 \%$ for the superonasal sector, $21.73 \%$ for the temporal sector and $4.34 \%$ for the inferonasal sector between the groups. This increased difference in the superior sector could probably be attributed to the arrangement of blood vessels on the disc.
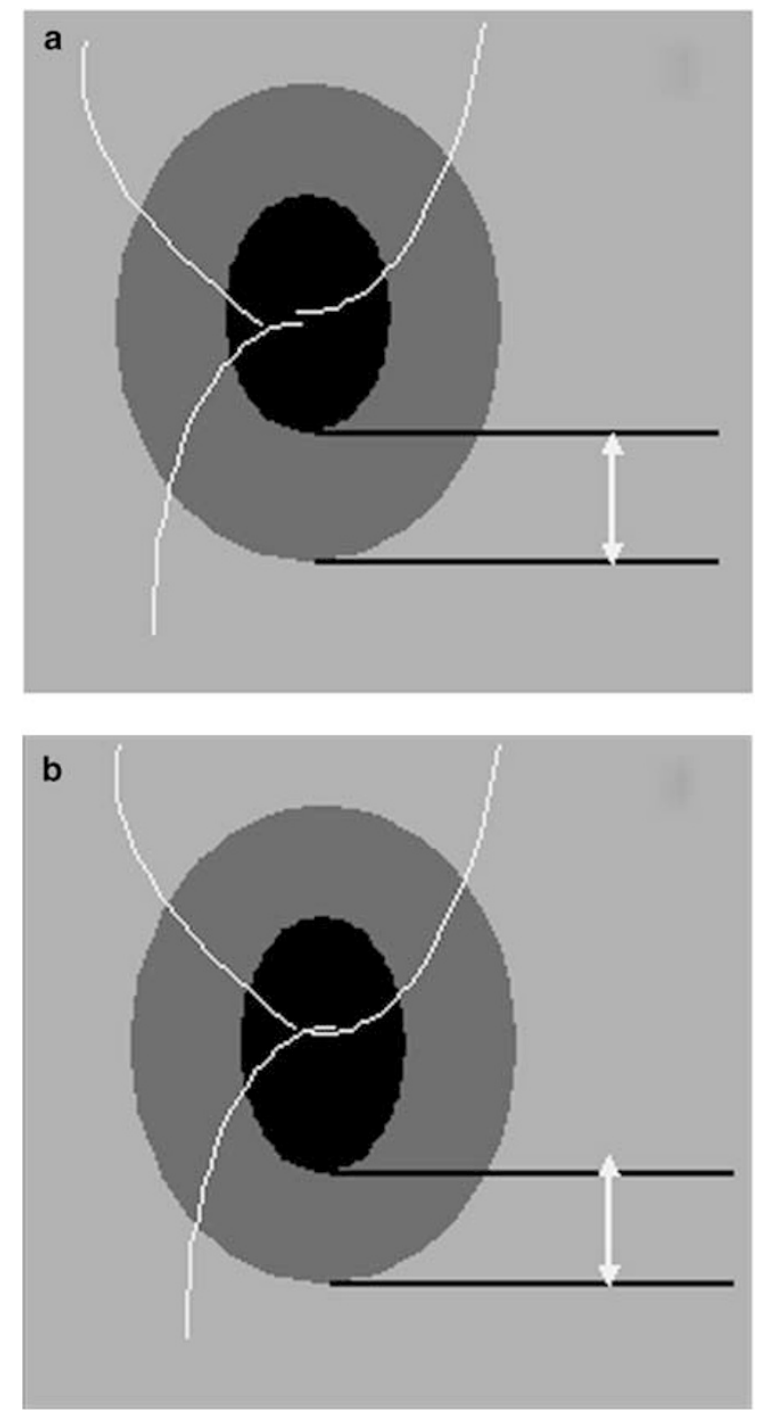

Figure $3(a, b)$ The loss of NRR as shown by the protruding arrowhead may be difficult to appreciate by slit lamp biomicroscopy.
In the present study, all individual rim sectors, as expected, were found to be significantly reduced in the glaucoma group. The highest sensitivity in detecting glaucoma patients from normal subjects was seen for the superotemporal and superonasal rim sectors followed by the inferotemporal and temporal disc sectors. The least change from control to glaucomatous eyes was seen in the inferonasal disc sector.

When the ratios of the rim areas were analysed, the ratio of the superonasal to inferonasal rim $(P=0.001)$ was found to have the highest significance between the two groups, followed by the ratio of the inferotemporal sector to the inferonasal rim sector $(P=0.003)$. This reflects the fact that the least change in the rim area was seen in the inferonasal sector. So, it would probably be justified to consider the above two ratios on HRT, in the diagnosis of early glaucoma in our population, rather than the ratio of the superior to the inferior sectors.

It is possible that racial differences in the morphology of the optic nerve head may be responsible for the pattern of NRR loss in our study population. The position of the central retinal vessel trunk in the lamina cribrosa has been suggested as an important factor in the pattern of NRR loss ${ }^{22}$ and that the sector of the optic disc that is furthest from the exit of the central vessel trunk out of the lamina cribrosa is the most susceptible to glaucomatous NRR loss.

None of values under the ROC (receiver operating curve) for the individual rim sectors or ratios of the sectors showed a significant diagnostic predictability in differentiating the eyes with glaucoma from normal eyes (Table 3). Among the parameters and ratios analysed, only two parameters were slightly significant - disc area and inferior to temporal ratio.

In the present study, sensitivity and specificity values using the NRR as the only parameter to identify glaucomatous optic neuropathy were low. One of the reasons may be that we studied only early glaucomatous damage. More importantly, analysis of a combination of variables in the form of a multivariate approach is known to improve the discrimination between normal and glaucomatous eyes. Most of the rim areas were statistically independent of optic disc size, except for the inferonasal rim area $(r=0.224)$. Considering the marked interindividual variation of the optic disc area, the independence of the rim areas is an advantage, because this would enable us to interpret directly the width ratios.

The analysis of perimetry in our patient sample showed superior field defects in $39.6 \%$, inferior field defects in $60.3 \%$, indicating that there was no preferential loss of NRR in the inferotemporal rim.

The major limitation of the present study is the small numbers of early glaucoma patients analysed. Another 
limitation is that we have analysed the ISNT rule by the quantitative values given by the HRT II, based on false colour HRT images, which include the retinal vessel trunk and large retinal vessels as part of the rim which would have influenced the results to some extent. While this may not be applicable in clinical evaluation since the definition of the cup by the HRT II and on biomicroscopic evaluation differ, we do have reports of good agreement between CSLO measurements and clinician estimates of vertical cup-disc ratios from stereoscopic photographs, particularly of patients with glaucoma. ${ }^{23}$

The present study has shown that in POAG patients in our population, the circular configuration of the disc with an almost equal NRR distribution probably leads to an almost equal diffuse loss of NRR superiorly as well as inferiorly with corresponding field defects. Also, while ratios of different rim sectors are statistically different in normal and early glaucoma eyes, there was poor diagnostic specificity and sensitivity in differentiating normal from early glaucoma eyes when only this parameter is used to diagnose glaucoma. Only inferior to temporal rim ratio seems to be marginally acceptable. Since the inferonasal rim sector showed the least loss of NRR, we suggest that the ratio of the NRR areas of the inferotemporal and superonasal sectors individually to the inferonasal sector is probably a better indicator of early glaucomatous loss than the ratio of the superior to inferior sector.

\section{References}

1 Betz PH, Camps F, Collignon-Brach J, Lavergne G Weekers R. Biometric study of the disc cup in open angle glaucoma. Graefes Arch Clin Exp Ophthalmol 1982; 218: 70-74.

2 Jonas JB, Mardin CY, Grundler AE. Comparison of measurements of neuroretinal rim area between confocal scanning laser tomography and planimetry of photographs. Br J Ophthalmol 1998; 82: 362-366.

3 Jonas JB, Gusek GC, Naumann GOH. Optic disc, cup and neuroretinal rim size, configuration, and correlation in normal eyes. Invest Ophthalmol Vis Sci 1988; 29: 1151-1154 (correction: Invest Ophthalmol Vis Sci 1991; 32 1893).

4 Tuulonen A, Airaksinen PJ. Initial glaucomatous optic disc and retinal nerve fiber layer abnormalities and their progression. Am J Ophthalmol 1991; 111: 485-490.

5 Quigley HA. II Changes in the appearance of the optic disc. Surv Ophthalmol 1985; 30: 117-126.

6 Pederson JE, Anderson DR. The mode of progressive disc cupping in ocular hypertension and glaucoma. Arch Ophthalmol 1980; 98: 490-495.
7 Jonas JB, Fernandez MC, Sturmer J. Pattern of glaucomatous neuroretinal rim loss. Ophthalmology 1993; 100: 63-68.

8 Hodapp E, Parrish IIRK, Anderson Dr. Clinical decisions in glaucoma. The CV Mosby: St Louis, 1993; 84-126.

9 Mikelberg FS, Wijsman K, Schulzer M. Reproducibility of topographic parameters obtained with the Heidelberg retina Tomograph. Glaucoma 1993; 2: 101-103.

10 Rohrschneider K, Burk ROW, Kruse FE, Volcker HE. Reproducibility of the optic nerve head topography with a new laser tomographic scanning device. Ophthalmology 1994; 101: 1044-1049.

11 Jonas JB, Budde WM, Lang P. Neuroretinal rim width ratios in morphological glaucoma diagnosis. Br J Ophthalmol 1998; 82: 1366-1371.

12 Jonas JB, Konigsreuther KA. Optic disc appearance in ocular hypertensive eyes. Am J Ophthalmol 1994; 117: 732-740.

13 Jonas JB, Burde WM. Is the nasal optic disc sector important for morphometric glaucoma diagnosis? $\mathrm{Br} J$ Ophthalmol 2002; 86: 1232-1235.

14 Mistlberger A, Liebmann JM, Greenfeld DS, Pons ME, Hoh ST, Ishikawa $\mathrm{H}$ et al. Heidelberg Retina Tomography and Optical Coherence Tomography in normal, ocular hypertensive and glaucomatous eyes. Ophthalmology 1999; 106: 2027-2032.

15 Medeiros FA, Zangwill LM, Bowd C, Weinreb RN. Comparison of the GDX VCC Scanning Laser Polarimeter, HRT II Confocal Scanning Laser Ophthalmoscope and Stratus OCT Optical Coherence Tomograph for the detection of glaucoma. Arch Ophthalmol 2004; 122: 827-837.

16 Agarwal HC, Gulati V, Sihota R. The normal optic nerve head on Heidelberg Retina Tomograph II Indian. J Ophthalmol 2003; 51: 25-33.

17 Jonas JB, Grundler AE. Optic disc morphology in juvenile primary open angle glaucoma. Graefes Arch Ophthalmol 1996; 234: $750-754$

18 Spaeth GL, Hitchings RA, Sivalingam E. The optic disc in glaucoma: pathogenetic correlation of five patterns of cupping in chronic open-angle glaucoma. Trans Am Acad Ophthalmol Otolaryngol 1976; 81: 217-223.

19 Tuulonen A. The morphological pattern of early glaucomatous damage. Curr Opin Ophthalmol 1993; 4: 29-34.

20 Airaksinen PJ, Drance SM, Douglas GR, Schulzer M, Wijsman K. Visual field and retinal nerve fiber layer comparisons in glaucoma. Arch Ophthalmol 1985; 103 205-207.

21 Mardin CY, Horn FK, Jonas JB, Budde WM. Perimetric glaucoma diagnosis by confocal scanning laser tomography of the optic disc. Br J Ophthalmol 1999; 83: 299-304.

22 Jonas JB, Fernandez MC. Shape of the neuroretinal rim and position of the central retinal vessels in glaucoma. $\mathrm{Br} \mathrm{J}$ Ophthalmol 1994; 78: 99-102.

23 Zangwill L, Shakiba S, Caprioli J, Weinreb RN. Agreement between clinicians and a confocal scanning laser ophthalmoscope in estimating cup/disc ratios. Am J Ophthalmol 1995; 119: 415-421. 